\title{
Static and Motional Feedthrough Capacitance of Flexural Microresonator
}

\author{
Wen-Teng Chang \\ Department of Electrical Engineering, National University of Kaohsiung, Kaohsiung 81148, Taiwan \\ Correspondence should be addressed to Wen-Teng Chang, wtchang@nuk.edu.tw
}

Received 16 June 2011; Accepted 8 August 2011

Academic Editor: Ching Liang Dai

Copyright () 2011 Wen-Teng Chang. This is an open access article distributed under the Creative Commons Attribution License, which permits unrestricted use, distribution, and reproduction in any medium, provided the original work is properly cited.

The present paper evaluates the static and motional feedthrough capacitance of a silicon carbide-based flexural-mode microelectromechanical system resonator. The static feedthrough capacitance was measured by a network analyzer under atmospheric pressure. The motional feedthrough was obtained by introducing various values into the modeling circuit in order to fit the Bode plots measured under reduced pressure. The static feedthrough capacitance was $0.02 \mathrm{pF}$, whereas the motional feedthrough capacitance of an identical device was about $0.2 \mathrm{pF}$, which is one order of magnitude larger than the static feedthrough capacitance.

\section{Introduction}

Microelectromechanical system (MEMS) resonators have attracted the radio frequency communication community's attention because of their excellent characteristics (Qs) $[1,2]$. Recently, several studies have analyzed and characterized electrical MEMS resonators in order to integrate them fully with an analog circuit. As a MEMS resonator is driven electrostatically, a motional current is generated based on the oscillating mass. Therefore, the resonator is modeled electrically with a series resistor, inductor, and capacitor connection when oscillating. The motional resistance is proportional to the energy dissipation, which is the reciprocal of the quality factor $[3,4]$. Lopez et al. showed that the fabricated driving gap can affect the motional resistance significantly [5]. In addition to motional components, the current also runs through an undesired path from the input to output ports modeled with a parallel feedthrough capacitance. Tanaka et al. showed that the feedthrough capacitance affects resonator performance and frequency response [6]. An accurate modeling or estimation is important in integrating the MEMS resonator into a circuit design, such as an oscillator and filter. However, parasitics are usually dependent on the substrate, layout and device manufacturing. The accurate estimation of parasitic capacitance is important but is difficult to extract from direct measurement [7]. Lee and Seshia successfully evaluated this capacitance for both low $Q$ and high $Q$ conditions [8]. The flexural-mode MEMS resonator suffers significantly from air damping in atmospheric pressure compared with that in a vacuum [9, 10]. Therefore, the present study can evaluate the lateral flexural resonator with a low $Q$ in atmospheric pressure and a high $Q$ in vacuum using an identical device. The proposed modeled electrical components from the MEMS resonator are based on the conclusion by Nguyen and Howe [1], which did not evaluate feedthrough capacitance yet. The feedthrough capacitance evaluation under static and oscillating modes can be obtained by direct measurement and curve fitting. Static feedthrough capacitance is accessible from direct measurement from network analyzer, while motional feedthrough capacitance is derived by curve fitting with the Bode plot under oscillation. The handy approach could enable a designer to evaluate the feedthrough capacitance of MEMS resonator for both static and motional statuses.

\section{Fabrication and Measurement Setup of MEMS Resonator}

The current work used silicon carbide as structural material because it has a high Young modulus which increases the acoustic velocity above that of silicon. The $3 \mathrm{C}$-silicon 


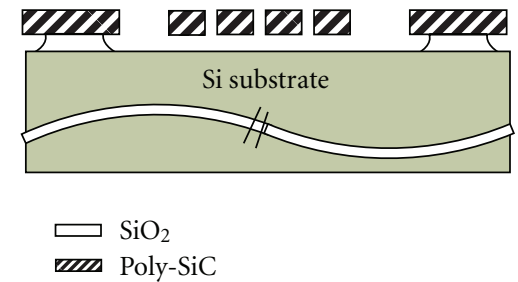

(a)

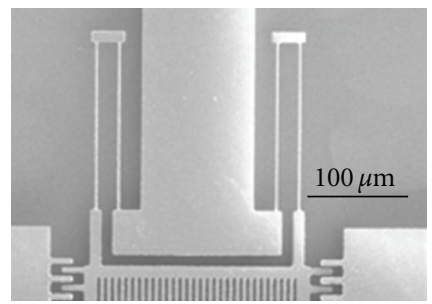

(b)

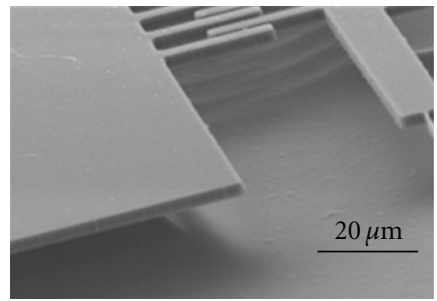

(c)

FigURE 1: (a) Schematic cross-sections of the 3C-SiC lateral resonators in the present study. (b) Top and (c) side views of the MEMS resonator.

(a)

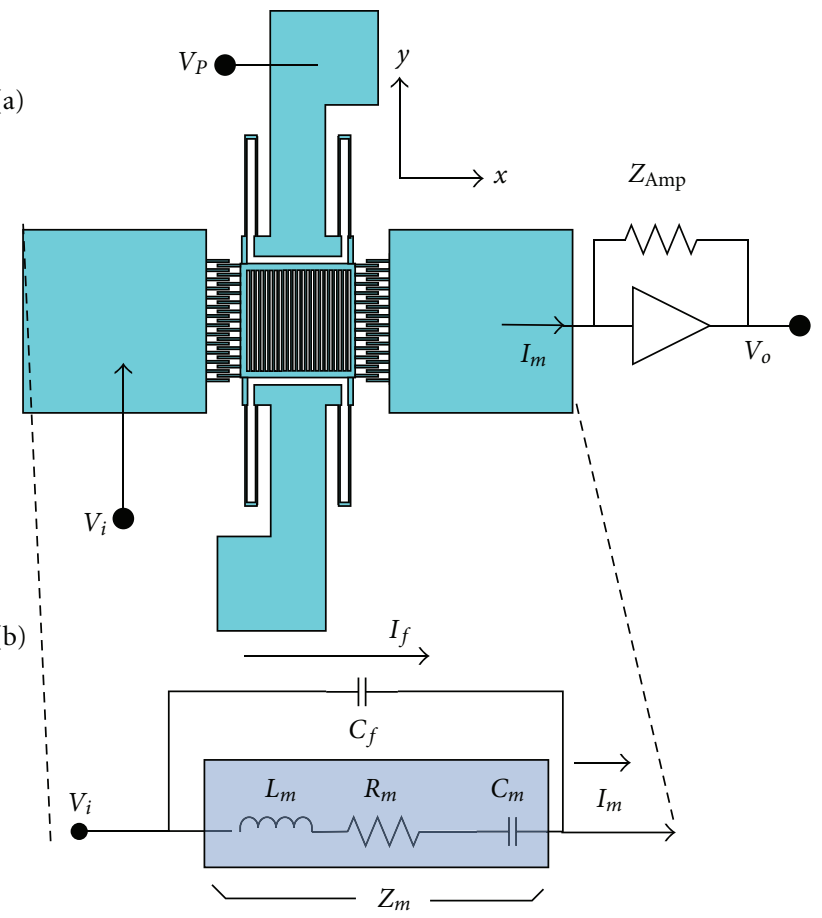

FIGURE 2: (a) Schematic of the experimental setup and (b) equivalent circuit of a MEMS resonator.

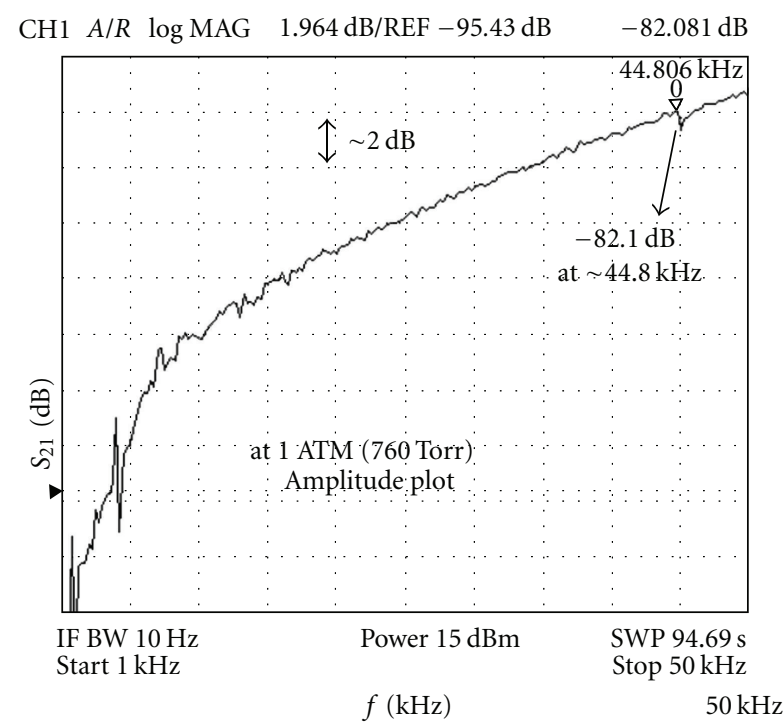

FIgURE 3: Magnitude plot of a flexural-mode resonator operated at atmospheric pressure. 


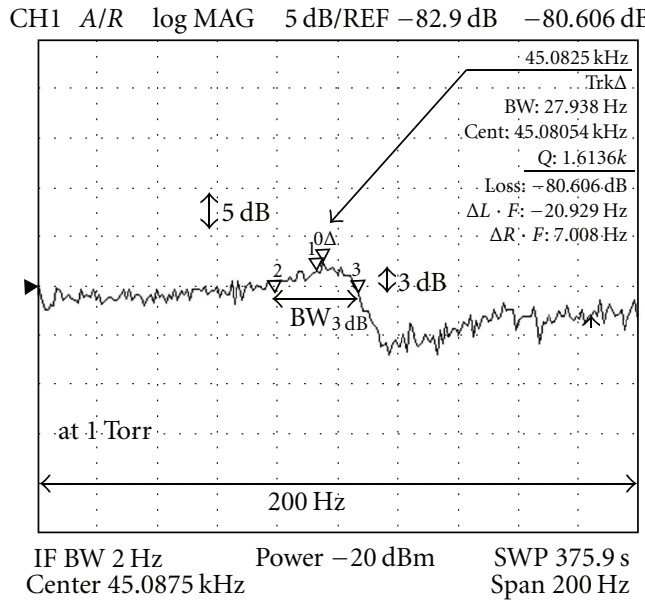

(a)

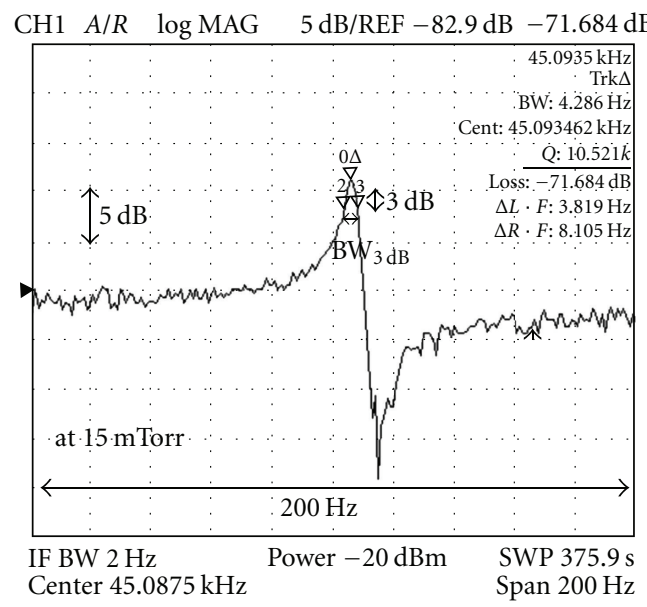

(c)

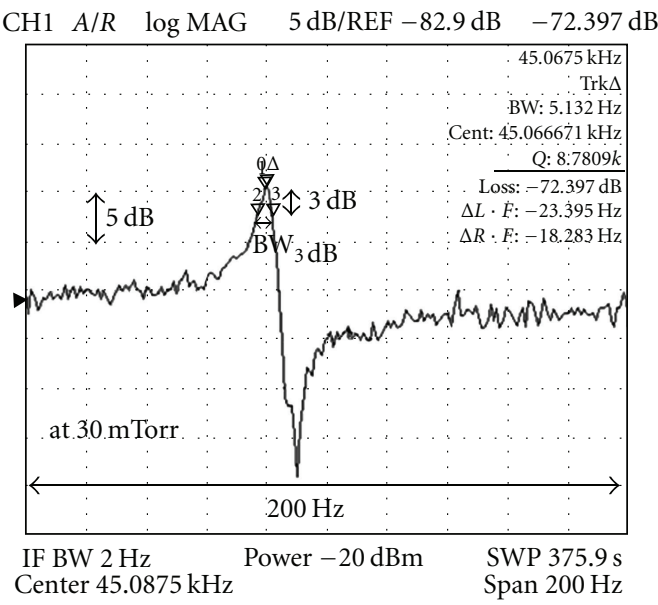

(b)

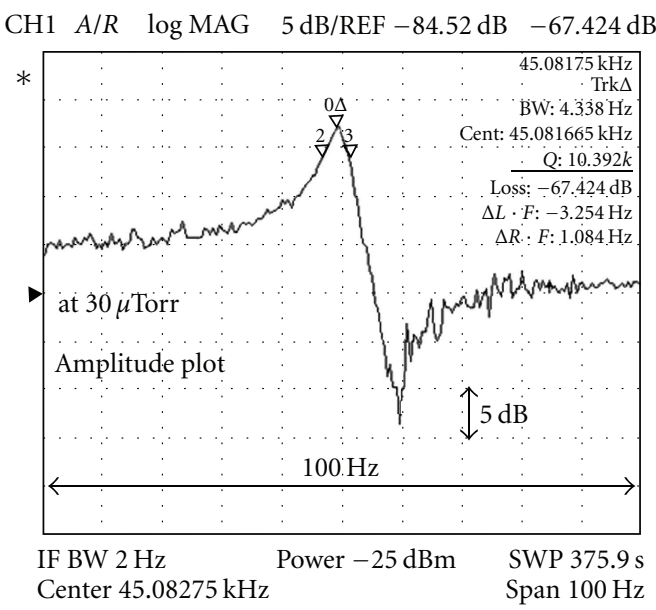

(d)

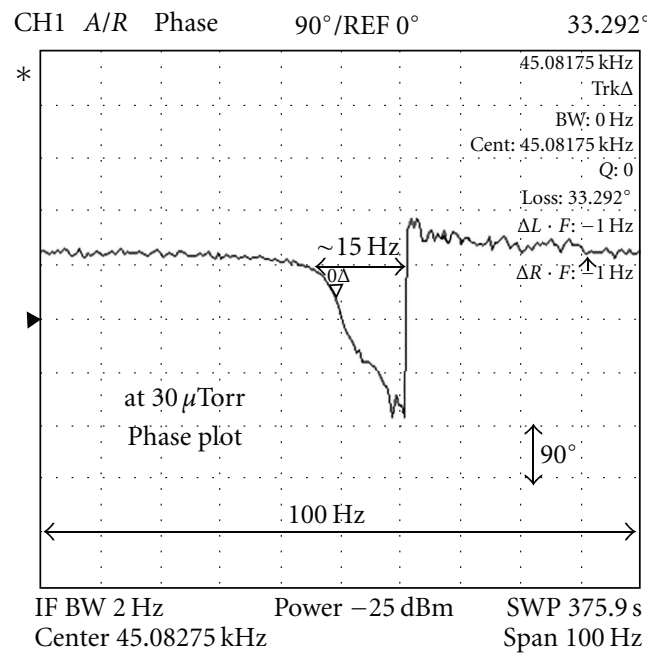

(e)

Figure 4: Magnitude plots of the flexural-mode resonator measured at (a) 1 torr, (b) 30 mtorr, (c) 15 mtorr, and (d) $30 \mu$ torr. (e) Phase plot of (d) under $30 \mu$ torr. 


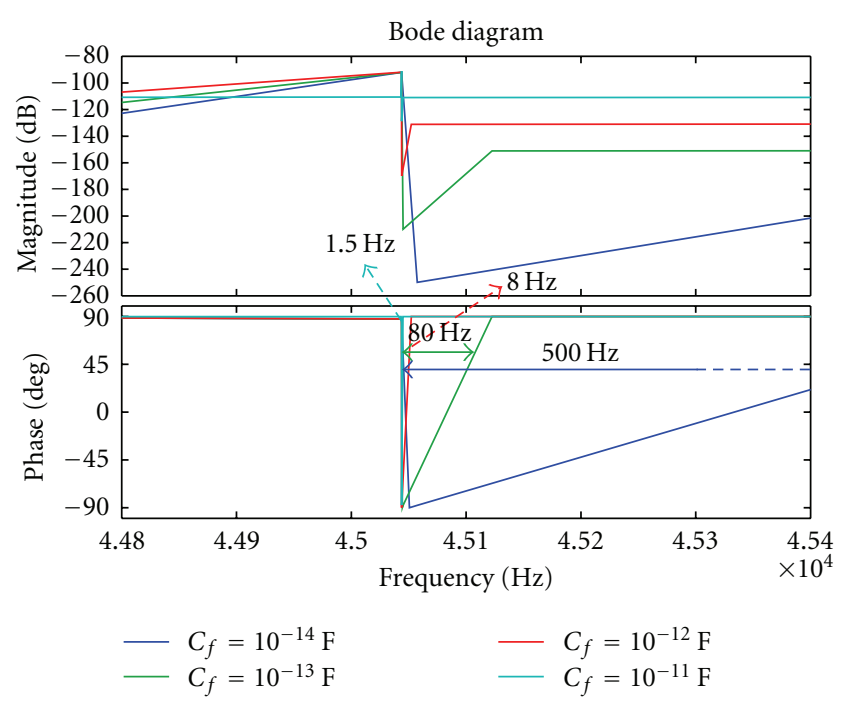

FIGURE 5: Simulated Bode diagrams for determining the appropriate feedthrough capacitance using values of $10^{-14}, 10^{-13}, 10^{-12}$, and $10^{-11} \mathrm{~F}$.

TABLE 1: Key parameters for the folded beam resonator used in determining motional components.

\begin{tabular}{lc}
\hline Parameters & \\
\hline$M:$ effective mass $(\mathrm{kg})$ & $9.74 \times 10^{-11}$ \\
$k:$ spring constant $(\mathrm{N} / \mathrm{m})$ & 7.81 \\
$V_{p}:$ bias voltage $(\mathrm{V})$ & 30 \\
$\partial C / \partial x:$ capacitance change of & $2.30 \times 10^{-10}$ \\
displacement $(\mathrm{F} / \mathrm{m})$ & 45081 \\
$f:$ resonant frequency $(\mathrm{Hz})$ & 10392 \\
$Q:$ quality factor & \\
\hline
\end{tabular}

TABle 2: Equivalent motional resistance $\left(R_{m}\right)$, capacitance $\left(C_{m}\right)$, and inductance $\left(L_{m}\right)$ of the resonator.

\begin{tabular}{ll}
\hline Motional components & \\
\hline$R_{m}(\mathrm{k} \Omega)$ & 1.54 \\
$C_{m}(\mathrm{aF})$ & 6.09 \\
$L_{m}(\mathrm{MH})$ & 2.05 \\
\hline
\end{tabular}

carbide resonators employed in the present work were developed originally from poly-SiC films deposited by lowpressure chemical vapor deposition (LPCVD). The details are pertaining to the fabrication and its use can be found in [4]. A series of resonators was fabricated from poly-SiC films deposited by LPCVD. The substrates comprised Si wafers capped with a thermally grown $1.5 \mu$ m-thick $\mathrm{SiO}_{2}$ film. Figure 1(a) shows the schematic cross-sections of the 3C-SiC lateral resonators used in the present study. Figures 1(b) and 1 (c) show the partial top and side views of the MEMS resonator, respectively. The fingers are well interdigitated on the proof mass and the electrode.

Figure 2(a) shows the schematic of the measurement setup of the MEMS resonator. One pad is utilized for the signal input, and the other is employed for the signal output.
The proof mass is suspended by four supporting beams connected to anchors. The resonator is driven with an input voltage $\left(V_{i}\right)$. A Philips SA5211 transimpedance amplifier is connected at the output to amplify the motional current with gain of $Z_{\mathrm{AMP}}$. When oscillating, the MEMS resonatorgenerated motional current is converted to a voltage output $\left(V_{o}\right)$ by the transimpedance amplifier. The transimpedance amplifier is chosen because it uses a bipolar junction transistor differential amplifier capable of providing a low noise output signal. In addition, the bandwidth reaches $180 \mathrm{MHz}$, which can satisfy the measurement range. Figure 2(b) shows the equivalent circuit of a MEMS resonator comprising an equivalent resistor $\left(R_{m}\right)$, capacitor $\left(C_{m}\right)$, inductor $\left(L_{m}\right)$, and feedthrough capacitor $\left(C_{f}\right)$. The feedthrough current $\left(I_{f}\right)$ can flow through due to carrier charge and discharge between ports and the shuttle (proof mass) even if the MEMS resonator is not oscillating. The motional current $\left(I_{m}\right)$ occurs only when the resonator is oscillating.

\section{Results and Discussion}

3.1. Modeling of an Oscillating MEMS Resonator. When an electrostatic actuation mechanism drives a MEMS resonator, motional current is generated based on the oscillating frequency. An oscillating MEMS resonator without feedthrough capacitance is modeled as an RCL circuit, denoted by $R_{m}, C_{m}$, and $L_{m}$, respectively. The values are modeled as [1]

$$
\begin{gathered}
C_{m}=\frac{\left[V_{P}(\partial C / \partial x)\right]^{2}}{k}, \\
L_{m}=\frac{k}{\omega_{r}^{2}\left[V_{p}(\partial C / \partial x)\right]^{2}}, \\
R_{m}=\frac{k}{\omega_{r} Q\left[V_{p}(\partial C / \partial x)\right]^{2}},
\end{gathered}
$$

where $\omega_{r}=\sqrt{k / m}, \mathrm{~V}_{P}$ is the bias voltage applied to amplify motional-current signals, $k$ is the spring constant, $\omega_{r}$ is the resonant angular velocity of the MEMS resonator, $\partial C / \partial x$ is the capacitance change per displacement, and $Q$ is the quality factor. However, considering the feedthrough capacitance $\left(C_{f}\right)$ from the input to the output port, leakage current occurs and can cause a $180^{\circ}$ phase shift at the oscillating frequency.

3.2. Measurement in Atmospheric Pressure. Air damping has been a significant issue for most flexural resonators, resulting in small signals and low-quality factors. For normal viscous damping, such as that in a flexural resonator under atmospheric pressure, the gas velocity is zero at the gas-surface interface. Figure 3 shows a typical magnitude plot of a flexural-mode resonator operated at atmospheric pressure, sweeping from 1 to $50 \mathrm{kHz}$. However, for the resonant peak, its $3 \mathrm{~dB}$ bandwidth is too small to be identified because of large air damping. Considering the feedthrough capacitance only, largely due to the gap between the proof mass and 
electrodes, as shown in Figure 2(a), the feedthrough current $\left(I_{f}\right)$ will be

$$
I_{f}=\frac{V_{i}}{\left(1 / j \omega C_{f}\right)},
$$

where $V_{i}$ is the input voltage applied from the network analyzer, $\omega$ is the sweep frequency of the radius, and $C_{f}$ is the feedthrough capacitance of the MEMS resonator from the input to the output port. Following the MEMS resonator, a transimpedance amplifier SA5211 was connected to the output voltage $\left(V_{o}\right)$ in the output port of the network analyzer. The feedthrough current is caused by a charge attraction between the electrodes and proof mass, or the shuttle

$$
V_{o}=I_{f} \cdot Z_{\mathrm{AMP}} \text {. }
$$

The transmission coefficient $S_{21}$ is extracted from (4) and (5). The coefficient is proportional to the sweep frequency beyond $10 \mathrm{kHz}$ sweeping. Although SA5211 is a $28 \mathrm{k} \Omega$ transimpedance amplifier with differential outputs, the gain becomes $14 \mathrm{k} \Omega\left(Z_{\mathrm{AMP}}\right)$ as grounding one of the outputs. Considering that $S_{21}=-82.1 \mathrm{~dB}$ at $44.8 \mathrm{kHz}$ and the amplification of gain; the static feedthrough capacitance is about $0.02 \mathrm{pF}$ from (6):

$$
S_{21}(\mathrm{~dB})=20 \log \left|\frac{V_{o}}{V_{i}}\right|=20 \log \left(2 \pi f C_{f} Z_{\mathrm{AMP}}\right) .
$$

3.3. Measurement in Reduced Pressures. Air damping comprises a significant part of the total energy dissipation in atmospheric pressure. The dissipation attributed to air damping is too high for the identification of the oscillation peak in Figure 3. For this viscous damping, the gas velocity is almost zero at the gas-surface interface, whereas the gas rarefaction at the interface occurs in reduced pressure. The resonant frequency shifts with the gas-rarefaction effect because of the change in the damping coefficient. Figures 4(a), 4(b), 4(c), and 4(d) show the magnitude plots of the flexuralmode resonator measured at 1 torr, 30 mtorr, 15 mtorr, and $30 \mu$ torr, respectively. Note that these plots have the same vertical and horizontal scales. The $Q s$ of the device reach sta-bility below 15 mtorr. In other words, the critical point for the folded beam resonator design is about 15 mtorr. Figure 4(e) shows the phase plot of Figure 4(d).

Using the measured parameters listed in Table 1, the equivalent motional resistance $\left(R_{m}\right)$, motional capacitance $\left(C_{m}\right)$, and motional inductance $\left(L_{m}\right)$ can be derived using (1), as listed in Table 2. Furthermore, by fitting the curve into the measured curve in Figures 4(d) and 4(e), the feedthrough capacitance $\left(C_{f}\right)$ of the circuit can be obtained. The modulus of the transfer function is

$$
\begin{aligned}
\frac{V_{o}(s)}{V_{i}(s)} & =\frac{Z_{\mathrm{AMP}}}{Z_{m}} \\
& =\frac{Z_{\mathrm{AMP}}}{\left(1 / s C_{f}\right) /\left(s L_{m}+R_{m}+1 / s C_{m}\right)} \\
& =\frac{Z_{\mathrm{AMP}}\left(1 / s C_{f}+1 / s C_{m}+R_{m}+s L_{m}\right)}{\left(1 / s C_{f}\right) \cdot\left(s L_{m}+R_{m}+1 / s C_{m}\right)},
\end{aligned}
$$

where $Z_{\mathrm{AMP}}$ is a constant $(14 \mathrm{k} \Omega)$, because the SA5211 provides a wide-band amplification within $180 \mathrm{MHz}, s$ is the frequency in complex number. Both of the numerator and the denominator parts of the fraction function in (7) become third order functions by multiplying $s^{2}$. Using the MATLAB transfer function, the simulated bandwidths for the phase from $90^{\circ}$ to $-90^{\circ}$ and then back to $90^{\circ}$ are $500,80,8$, and $1.5 \mathrm{~Hz}$ for $10^{-14}, 10^{-13}, 10^{-12}$, and $10^{-11} \mathrm{~F}$ feedthrough capa-citances, respectively, as shown in Figure 5. In analyzing this phase shift, this motional feedthrough capacitance is $2 \times 10^{-13} \mathrm{~F}$, or $0.2 \mathrm{pF}$, which is about one order of magnitude larger than the static feedthrough capacitance.

\section{Conclusion}

The present study evaluated static and motional feedthrough capacitance. The results show that motional feedthrough capacitance is roughly one order of magnitude larger than that in static mode. Some precise RCL meters may also be useful in obtaining static feedthrough capacitance, although the current work used characterization curve to find the static feedthrough capacitance. Additionally, other modes besides the flexural-mode MEMS resonator would be suitable for determining motional feedthrough capacitance by fitting the Bode plot curves.

\section{Acknowledgment}

This paper was supported by the National Science Council under Grant no. 96-2218-E-390-001.

\section{References}

[1] C. T. C. Nguyen and R. T. Howe, "Integrated CMOS micromechanical resonator high-Q oscillator," IEEE Journal of SolidState Circuits, vol. 34, no. 4, pp. 440-455, 1999.

[2] J. F. Gong, Z. Y. Xiao, and P. C. H. Chan, "Integration of an RF MEMS resonator with a bulk CMOS process using a lowtemperature and dry-release fabrication method," Journal of Micromechanics and Microengineering, vol. 17, no. 1, article 003, pp. 20-25, 2007.

[3] W. T. Chang, "Clamping losses of folded- and straight-beam MEMS resonators made from polycrystalline 3C-SiC films," International Journal Modern Physics B, vol. 25, no. 6, pp. 885896, 2011.

[4] W. T. Chang and C. Zorman, "Grain size control of (111) polycrystalline $3 \mathrm{C}$-SiC films by doping used as folded-beam MEMS resonators for energy dissipation," Microsystem Technologies, vol. 15, no. 6, pp. 875-880, 2009.

[5] J. L. Lopez, J. Verd, J. Teva et al., "Integration of RF-MEMS resonators on submicrometric commercial CMOS technologies," Journal of Micromechanics and Microengineering, vol. 19, no. 1, Article ID 015002, 2009.

[6] K. Tanaka, R. Kihara, A. Sánchez-Amores, J. Montserrat, and J. Esteve, "Parasitic effect on silicon MEMS resonator model parameters," Microelectronic Engineering, vol. 84, no. 5-8, pp. 1363-1368, 2007.

[7] G. Zhu, J. Penet, and L. Saydy, "Modeling and control of electrostatically actuated MEMS in the presence of parasitics and parametric uncertainties," Journal of Dynamic Systems, Measurement and Control, vol. 129, no. 6, pp. 786-794, 2007. 
[8] J. E.-Y. Lee and A. A. Seshia, "Direct parameter extraction in feedthrough-embedded capacitive MEMS resonators," Sensors and Actuators, A, vol. 167, no. 2, pp. 237-244, 2011.

[9] X. Zhang and W. C. Tang, "Viscous air damping in laterally driven microresonators," Proceedings of the IEEE Micro Electro Mechanical Systems, pp. 199-204, 1994.

[10] M. Bao and H. Yang, "Squeeze film air damping in MEMS," Sensors and Actuators, A, vol. 136, no. 1, pp. 3-27, 2007. 

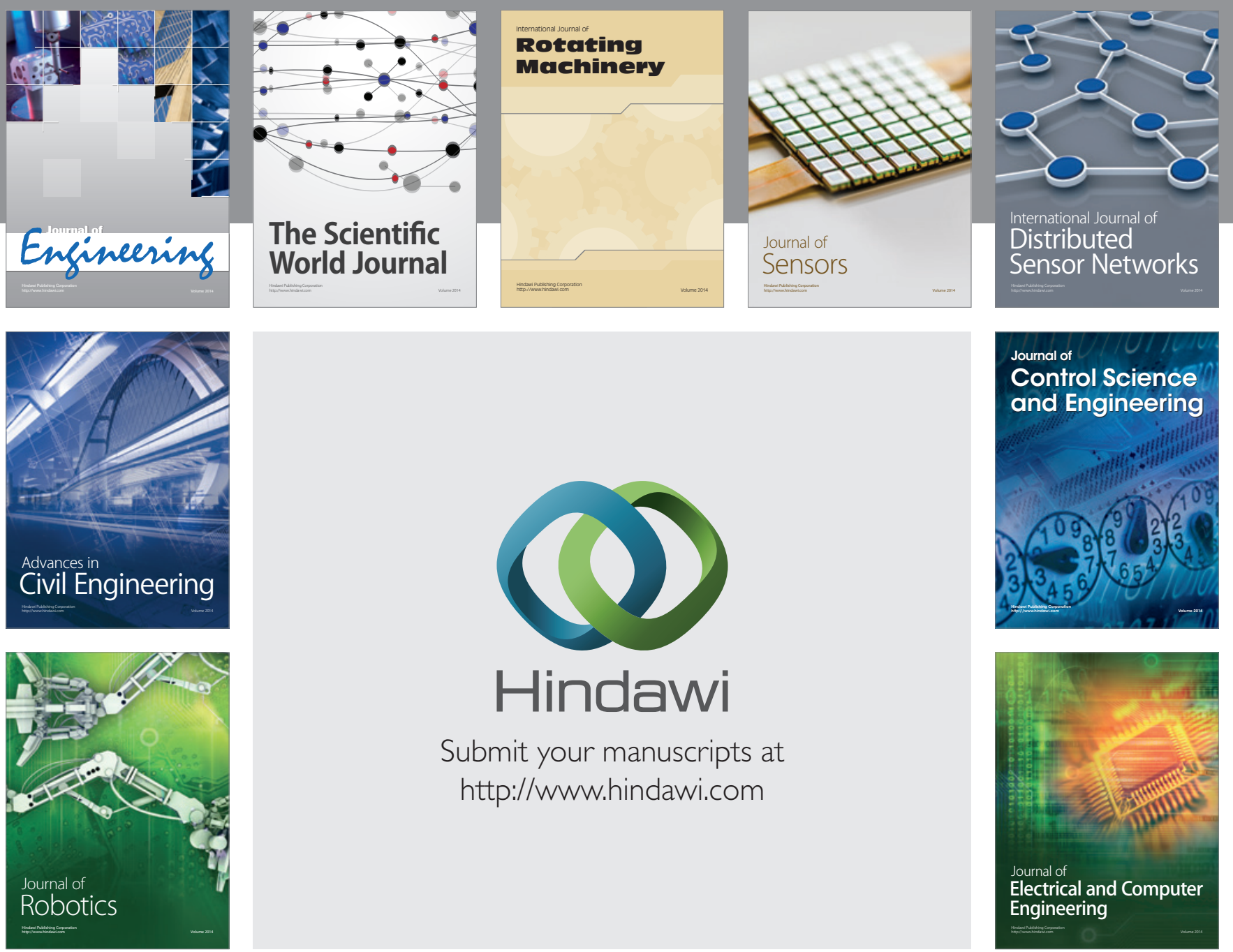

Submit your manuscripts at

http://www.hindawi.com
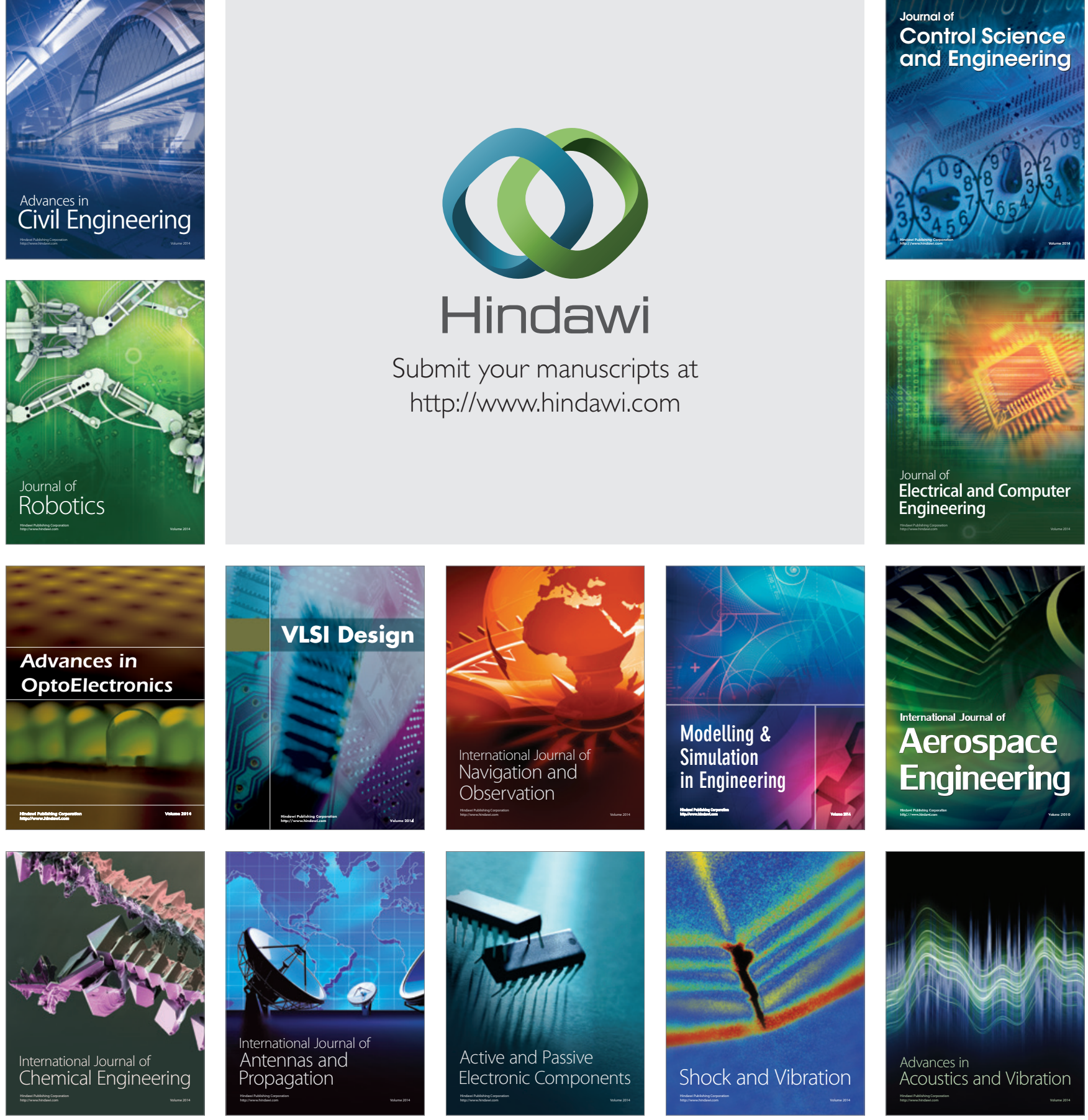